\title{
Bedtime routine (child)
}

\section{George Kitsaras ${ }^{1}$}

1 The University of Manchester

Potential competing interests: The author(s) declared that no potential competing interests exist.

Series of activities that take place roughly the hour before a child goes to bed. Activities should be fairly consistent over the week including the weekend. Activities include parent-child interactions and a series of behaviours that build up the routine each night. 